\title{
First recombinant Factor XIII approved
}

The US Food and Drug Administration (FDA) approved in December Bagsværd, Denmarkbased Novo Nordisk's Tretten (catridecacog), the first recombinant product to prevent bleeding in patients with the very rare autosomal recessive clotting disorder, congenital Factor XIII A-subunit deficiency. Despite its predicted high cost, Tretten is expected to rapidly supersede its plasma-derived counterpart.

The A subunit of Factor XIII is the terminal transglutaminase enzyme in the clotting cascade and plays a critical role in hemostasis. It catalyzes the cross-linking of fibrin and other coagulation proteins into a strong fibrin mesh that stabilizes blood clots. A deficiency in Factor XIII leads to unstable clots that eventually break down, increasing the risk of serious and life-threatening bleeding.

Tretten is a recombinant analog of Factor XIII A-subunit produced in yeast cells. During clinical development, 77 patients with congenital Factor XIII A-subunit deficiency were infused with Tretten, which was effective in preventing bleeding in $90 \%$ of patients when given monthly, according to the FDA.

Tretten is expected to appeal to patients who have typically been treated with Corifact, a Factor XIII concentrate derived from pooled human plasma from CSL Behring, King of Prussia, Pennsylvania, and approved by the
FDA in February 2011. Despite being heat treated, Corifact carries a risk of transmitting viruses and the Creutzfeldt-Jakob disease agent.

Pricing could be decisive. Plasma-derived products tend to be much cheaper than recombinant versions. Tretten is likely to cost around $\$ 400,000$ in the US, in line with other recombinant, prophylactic regimens in hemophilia, according to Karen Andersen, senior biotech analyst at Morningstar in Chicago. "Overall, recombinant proteins like Tretten are preferred over plasma-derived counterparts, as theoretically there is still the possibility of transmitting infectious diseases through plasma," says Andersen. In the larger hemophilia A market, she points out, switching to recombinant products was fairly rapid, once they became available.

Earnings from Tretten are not expected to be substantial, however. Factor XIII A-subunit deficiency is estimated to occur in only 1 in 3 million to 5 million births-that's just 108 people in the US and 1,054 worldwide. Andersen says peak sales of $\$ 200$ million for the entire market would be aggressive, and others suggest $\$ 84$ million is more likely. Additionally, Novo Nordisk must pay an undisclosed royalty on Tretten sales to Bristol-Myers Squibb of New York. In 2004, Novo licensed worldwide rights to the

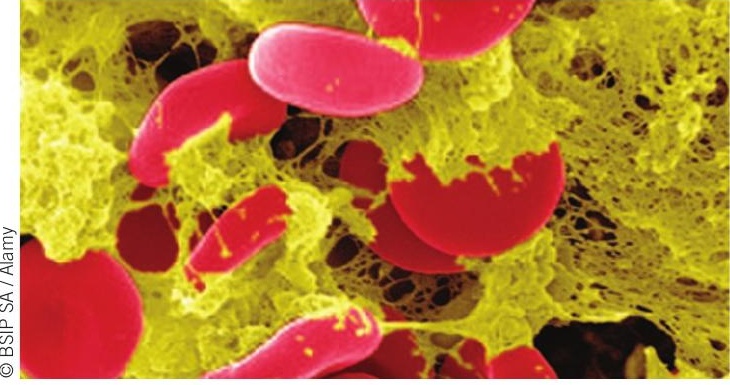

Factor XIII deficiency leads to unstable clots that eventually break down, increasing the risk of spontaneous hemorrhage.

recombinant Factor XIII portfolio of ZymoGenetics of Seattle, which Bristol-Myers Squibb subsequently acquired in 2010 .

Although Tretten is too small to make a difference for the equity markets, says Carsten Lønborg Madsen, company analyst at Carnegie Investment Bank Copenhagen, the product may support Novo's ambitions to become a dedicated player within bleeding disorders as it will help the company to offer a full range of treatments. Tretten, which was approved in Canada and, as NovoThirteen, in the EU, Switzerland and Australia in 2012, is expected to launch in the US during the next few months.

\section{Around the world in a month}

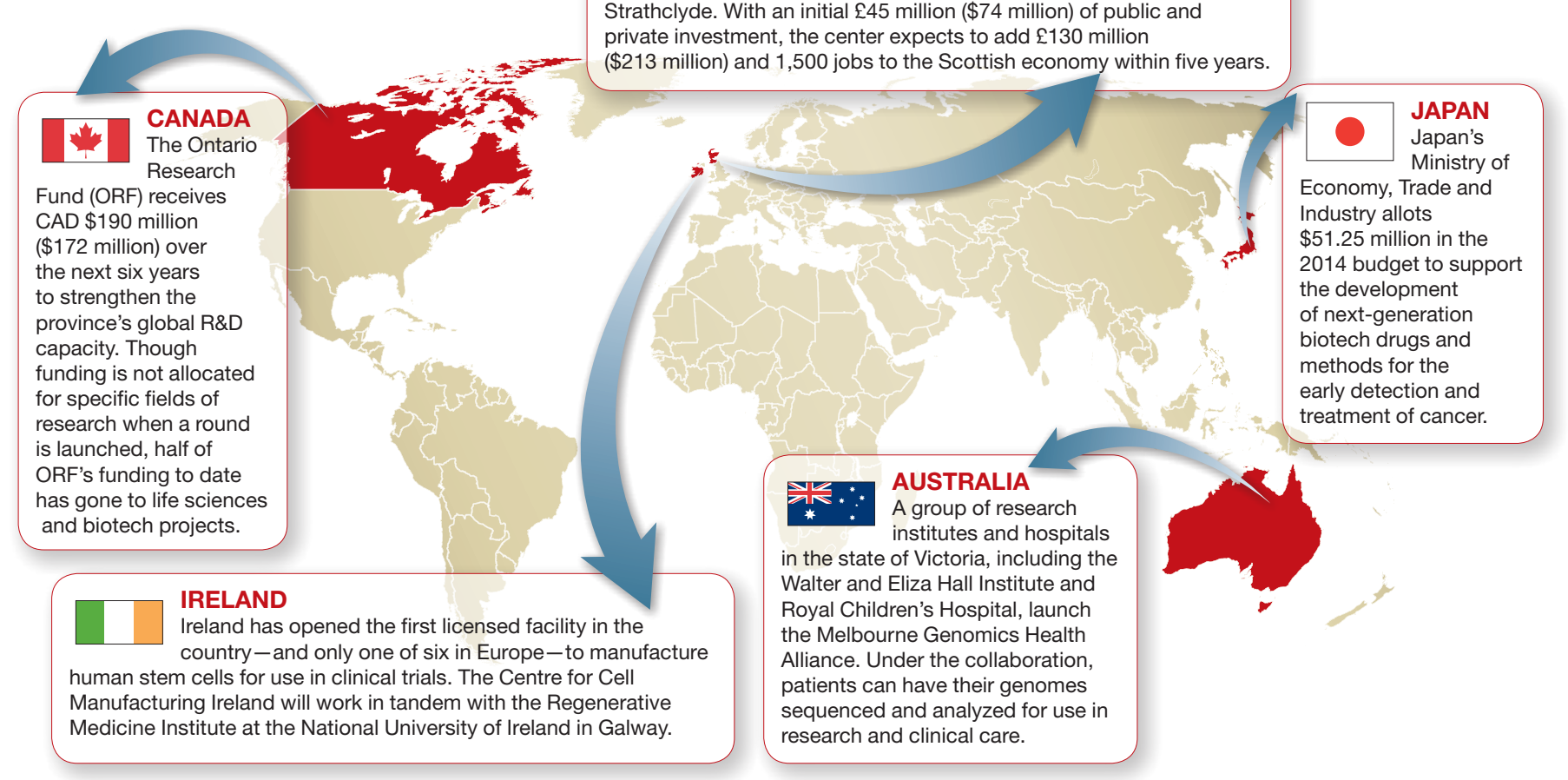

